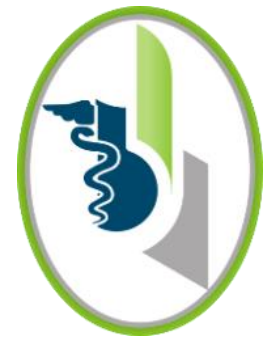

ACCESO ABIERTO

Para citaciones: Garcia, Y., Filott, M., Campo, M., Gomez, L., Bettin, A. (2020). Perfiles de los fenotipos de resistencia en Escherichia coli y Klebsiella pneumoniae en Barranquilla, Colombia. Revista Ciencias Biomédicas, 9(1), 15 24.

Recibido: 24 de julio de 2019 Aprobado: 18 de octubre de 2019

Autor de correspondencia: Alfonso Bettin Martínez abettin@unimetro.edu.co

Editor: Inés Benedetti. Universidad de Cartagena-Colombia.

\section{Perfiles de los fenotipos de resistencia en Escherichia coli y Klebsiella pneumoniae en Barranquilla, Colombia.}

\author{
Resistance phenotype profiles in Escherichia coli and Klebsiella \\ pneumoniae in Barranquilla, Colombia.
}
Yina García Toscano ${ }^{1,2}$, Margarita Filott Támara ${ }^{1,2}$, Mirna Campo Urbina ${ }^{1}$, Lucila Gómez Rodriguez ${ }^{1}$ Alfonso Bettín Martínez (1,3

${ }^{1}$ Grupo Caribe de Investigación en Enfermedades de tipo Infeccioso y Resistencia Microbiana.

Universidad Metropolitana, Barranquilla.

${ }^{2}$ Programa de Bacteriología, Universidad Metropolitana, Barranquilla.

${ }^{3}$ Departamento de Medicina. Universidad del Norte, Barranquilla.

\section{RESUMEN}

Introducción: La aparición y rápida diseminación de Escherichia coli (E. coli) y Klebsiella pneumoniae (K. pneumoniae) resistentes a cefalosporinas de tercera generación se considera como un problema de salud pública a nivel mundial.

Objetivo: determinar el perfil de susceptibilidad de E. coli y $K$. pneumoniae resistentes a cefalosporinas de tercera generación de cuatro instituciones de salud de la ciudad de Barranquilla, Colombia.

Métodos: estudio descriptivo de corte transversal. Se tomaron registros de 1250 aislamientos ( 835 E. coli y $415 \mathrm{~K}$. pneumoniae) productores de beta-lactamasas de espectro extendido (BLEE) por método de concentración inhibitoria mínima, reportados por las instituciones participantes. Se analizó una muestra por conveniencia de 100 cepas de E. coli y 64 de K. pneumoniae. Los aislamientos se clasificaron en perfiles de resistencia: perfiles (I - III) de E. coli y perfiles (I - IV) de K. pneumoniae, indicando resistencia a otros antibióticos beta-lactámicos y a otras familias de antibióticos.

Resultados: la prevalencia de E. coli y $K$. pneumoniae productoras de BLEE fue $23,5 \%$ y $26,7 \%$. Los servicios afectados fueron urgencias (32 y 12,5\%), consulta externa (31 y 7,81\%), hospitalizados (28 y 46,87\%) y cuidados intensivos (9 y $32,81 \%$ ) respectivamente. Se encontró alta sensibilidad a carbapenémicos, piperacilina-tazobactam, cefoxitin y cefepime. Alta resistencia a trimetoprimsulfametoxazol y ciprofloxacina.

Conclusiones: los perfiles fenotípicos de BLEE circulantes en los servicios hospitalarios de instituciones de Barranquilla se acompañan de multirresistencia a cefepime, trimetoprim-sulfametoxazol, ciprofloxacina y gentamicina, sin embargo, aún mantienen alta sensibilidad a los carbapenemes, piperacilina-tazobactam y amikacina.

Palabras Clave: Escherichia coli; Klebsiella pneumoniae; cefalosporinas de tercera generación; carbapenémicos; resistencia bacteriana; beta-lactamasas. 


\begin{abstract}
Introduction: The appearance and rapid spread of E. coli and $K$. pneumoniae resistant to third-generation cephalosporins is within the biggest threats to global health.
\end{abstract}

Objective: to determine the susceptibility profile of third-generation cephalosporins resistant E. coli and $K$. pneumoniae isolates recovered in four health institutions of Barranquilla city, Colombia.

Methods: a cross-sectional descriptive study. Records were taken of 1250 isolates (835 E. coli and $415 \mathrm{~K}$. pneumoniae) producers of extended-spectrum betalactamases (ESBL) by the minimum inhibitory concentration method, reported by participating institutions. A sample was analyzed for convenience of 100 strains of $E$. coli and 64 of $K$. pneumoniae. The isolates were classified into resistance profiles: profiles (I - III) of E. coli and profiles (I - IV) of K. pneumoniae, indicating resistance to other beta-lactam antibiotics and other families of antibiotics.

Results: the prevalence of ESBL producing E. coli and K. pneumoniae was $23.5 \%$ and $26.7 \%$. The affected services were emergencies (32 and 12.5\%), outpatient consultation (31 and 7.81\%), hospitalized (28 and 46.87\%) and intensive care ( 9 and $32.81 \%$ ). High sensitivity to carbapenems, piperacillin-tazobactam, cefoxitin and cefepime was found. High resistance to trimethoprim- sulfamethoxazole and ciprofloxacin.

Conclusion: the phenotypic profiles of ESBL circulating in the hospital services of institutions in Barranquilla are accompanied by multi-resistance to cefepime, trimethoprim-sulfamethoxazole, ciprofloxacin and gentamicin, however, they still have high sensitivity to carbapenems, piperacillin-tazobactam and amikacin.

Keywords: Escherichia coli; Klebsiella pneumoniae; third-generation cephalosporins; carbapenems; antimicrobial resistance; beta-lactamases.

\section{INTRODUCCIÓN}

La aparición y rápida diseminación de microorganismos como Escherichia coli (E. coli) y Klebsiella pneumoniae (K. pneumoniae) resistentes a cefalosporinas de tercera generación (cefotaxima, ceftazidima y ceftriaxona) se considera un problema de salud pública a nivel mundial (1-4). La resistencia a estos, está dada en gran parte por la producción de $\beta$-lactamasas de espectro extendido (BLEE), enzimas transportadas por elementos genéticos móviles, los cuales a su vez albergan genes de resistencia a otras familias de antibióticos (aminoglucósidos, fluoroquinolonas y trimetoprimsulfametoxazol). Este fenómeno retrasa el inicio de un tratamiento apropiado, aumenta la estancia hospitalaria y las tasas de morbilidad y mortalidad, generando un costo elevado en la atención sanitaria
(5-8). Además, limita las opciones terapéuticas para bacterias productoras de $\operatorname{BLEE}(1,9)$, un aspecto importante en las unidades de cuidados intensivos (UCI) (10). Esto ha llevado a que exista un gran interés por parte de redes y grupos para participar en la prevención, vigilancia y control de la resistencia a los antimicrobianos, un recurso importante $\mathrm{y}$ limitado para el tratamiento de infecciones producidas por bacilos Gram negativos $(11,12)$.

A nivel mundial, los centros para el control y la prevención de enfermedades de los Estados Unidos clasifican a las enterobacterias resistentes a las cefalosporinas de tercera generación como una amenaza grave, indicando que cada año en este país, aproximadamente dos millones de personas adquieren infecciones graves ocasionadas por bacterias resistentes y más de 23,000 personas 
mueren producto de estos microorganismos $(4,13)$. En América Latina, las tasas de infecciones asociadas a la atención en salud (IAAS) causadas por enterobacterias productoras de BLEE han aumentado desde el 2005 hasta un 32\% en E. coli y hasta el $58 \%$ en $\mathrm{K}$. pneumoniae, tasas más altas que en otras regiones del mundo (12). En Colombia, la resistencia a cefalosporinas de tercera y cuarta generación muestra variabilidad en los reportes, dependiendo de la región, localización hospitalaria, sitio de infección y uso de antimicrobianos $(14,15)$. Según el Instituto Nacional de Salud (INS), en el 2018, el distrito de Barranquilla había superado la media nacional de resistencia a cefalosporinas de tercera generación en los servicios de UCI y No UCI tanto para E. coli como para K. pneumoniae, con valores promedios de $35,8 \%$ y $28 \%$ respectivamente; sin embargo, estos porcentajes fueron calculados sobre datos parcializados y con bajo número de aislamientos, considerando que la información de las Unidades Primarias Generadoras de Datos (UPGD) de la región Caribe es limitada según este reporte (16). Con base en lo anterior, en este estudio se determinaron los perfiles de susceptibilidad de E. coli y K. pneumoniae resistente a cefalosporinas de tercera generación en cuatro instituciones de salud de la ciudad de Barranquilla, Colombia, con el fin de monitorear el comportamiento de estas bacterias multiresistentes.

\section{MÉTODOS}

\section{Tipo de estudio}

Se realizó un estudio observacional descriptivo de corte transversal, de marzo de 2017 a marzo de 2018.

\section{Obtención de la información}

Durante el período de estudio se obtuvo información de cuatro instituciones de salud de la ciudad de Barranquilla, Colombia, en las cuales el número total de aislamientos de E. coli y K. pneumoniae fue de 3560 y 1554 respectivamente (Tabla 1). De esta población, 1250 aislamientos (835 de E. coli y 415 de K. pneumoniae) fueron reportados como resistentes a una o varias cefalosporinas de tercera generación (cefotaxima, ceftazidima y ceftriaxona) según el método de concentración inhibitoria mínima (CIM) (17, 18). La identificación y detección de la susceptibilidad a los antibióticos se realizó por Microscan ${ }^{\circledR}$ (Siemens), Phoenix ${ }^{\circledR}$ (Beckton Dickinson) y Vitek ${ }^{\circledR}$ (BioMérieux). Las cepas ATCC® 25922 y ATCC® 700603 fueron utilizadas como control interno.

\section{Caracterización de los aislamientos}

De los 835 aislamientos de E. coli y 415 de K. pneumoniae reportados como resistentes a cefalosporinas de tercera generación, 164 aislamientos (100 de E. coli y 64 de K. pneumoniae) fueron seleccionados por conveniencia y enviados para su caracterización fenotípica al laboratorio de Biología Molecular de la Universidad Metropolitana, Barranquilla. Solo se tuvo en cuenta, el primer aislamiento de cada paciente (19). Los aislamientos fueron clasificados en cuatro categorías de acuerdo con el servicio de procedencia: consulta externa, urgencias, UCI y hospitalizados. Además, con base a los resultados de la CIM reportada para cefalosporinas de tercera generación, los aislamientos se clasificaron en perfiles fenotípicos de resistencia: perfiles (I - III) de E. coli y perfiles (I - IV) de K. pneumoniae. Estos perfiles agrupan resultados de la prueba de doble disco, resistencia a otros $\beta$-lactámicos y la resistencia a otras familias de antibióticos.

\section{Caracterización fenotípica}

La detección fenotípica de BLEE en los 164 aislamientos se realizó por medio de la prueba confirmatoria de doble disco (D-D) siguiendo los lineamientos sugeridos por el CLSI $(17,18)$. Las placas de agar Mueller Hinton (Beckton Dickinson) fueron inoculadas con cada aislamiento y se colocaron discos de cefotaxima $(30 \mu \mathrm{g})$, cefotaximaclavulanico $(30 / 10 \mu \mathrm{g})$, ceftazidima $(30 \mu \mathrm{g}) \mathrm{y}$ ceftazidima-clavulanico $(30 / 10 \mu \mathrm{g})$. Se consideraron cepas productoras de BLEE cuando la diferencia en los halos de inhibición fue mayor o igual a 5 milímetros (mm) (20). Teniendo en cuenta los resultados de la prueba de doble disco, se analizó si un resultado positivo se correlacionaba directamente con los valores de CIM reportados. 


\section{Análisis estadístico}

Se creó una base de datos mediante una hoja de cálculo de Excel en la que se registraron los resultados de las pruebas de susceptibilidad e información suministrada por el laboratorio clínico de las instituciones participantes en el estudio. Se realizó un análisis descriptivo que incluyó la distribución de porcentajes y frecuencias de susceptibilidad de las dos especies estudiadas y gráficos de dispersión utilizados para el análisis de la CIM. Todos los análisis fueron realizados a través del software estadístico Statgraphics, (Statgraphics Technologies, Inc).

\section{RESULTADOS}

La prevalencia promedio de resistencia a cefalosporinas de tercera generación reportada por las instituciones participantes fue $23,5 \%$ para $\mathrm{E}$. coli y $26,7 \%$ para K. pneumoniae (Tabla 1). De los 100 aislamientos recuperados de E. coli, el $69 \%$ provenían de orina, $16 \%$ de sangre, y $15 \%$ de otros tipos de muestras. En cuanto a su distribución por servicio hospitalario, E. coli se recuperó en un $32 \%$ de urgencias, $31 \%$ de consulta externa, $28 \%$ de hospitalizados y $9 \%$ de UCI.

En el caso de los 64 aislamientos recuperados de K. pneumoniae, el 39,1\% provenía de sangre, $23,4 \%$ de orina, $12,5 \%$ de muestras respiratorias y $25 \%$ de otros tipos de muestras. Se recuperaron $46,87 \%$ de hospitalizados, $32,81 \%$ de UCI, $12,5 \%$ de urgencias y $7,81 \%$ de consulta externa.
En cuanto a los valores de CIM resistentes a las cefalosporinas, el $98 \%$ de E. coli presento una CIM a cefotaxima entre $32 \mu \mathrm{g} / \mathrm{mL}$ y $64 \mu \mathrm{g} / \mathrm{mL}$, y un $95 \%$ para ceftazidima entre $4 \mu \mathrm{g} / \mathrm{mL}$ y $16 \mu \mathrm{g} / \mathrm{mL}$. Para K. pneumoniae, la cefotaxima osciló entre $4 \mu \mathrm{g} / \mathrm{mL}$ y $64 \mu \mathrm{g} / \mathrm{mL}$, concentrándose el mayor número de aislamientos $(56,25 \%)$ en CIM de $32 \mu \mathrm{g} / \mathrm{m}$. Para ceftazidima la CIM fue de $16 \mu \mathrm{g} / \mathrm{mL}$ en el $71,87 \%$ de los casos. Tanto E. coli como K. pneumoniae presentaron CIM entre $1 \mu \mathrm{g} / \mathrm{mL}$ y $16 \mu \mathrm{g} / \mathrm{mL}$ en el caso de cefepime (Figura 1).

Referente a los porcentajes de susceptibilidad frente a otros antibióticos $\beta$-lactámicos: el 100\% de E. coli fue sensible a meropenem, $95 \%$ a ertapenem, $88 \%$ a piperacilina-tazobactam, $66 \%$ a cefoxitin y $18 \%$ a cefepime, En el caso de K. pneumoniae, el 93,8\% fue sensible a meropenem, $81,2 \%$ a ertapenem, 79,7\% a cefoxitin, $60,9 \%$ a piperacilina-tazobactam (2/4 $\mu \mathrm{g} / \mathrm{mL}-8 / 4 \mu \mathrm{g} / \mathrm{mL}$ ) y $28,0 \%$ al cefepime (CIM entre $1 \mu \mathrm{g} / \mathrm{mL}$ y $8 \mu \mathrm{g} / \mathrm{mL})$.

Por otra parte, se observó que más del $80 \%$ de E. coli positiva para BLEE fue resistente a trimetoprimsulfametoxazol y a ciprofloxacina; de estas el 71,4\% provenían de muestras de orina. A su vez, K. pneumoniae tuvo resistencia a estos mismos antibióticos en $61 \%$ y 78,1\%, respectivamente. Respecto a la resistencia a gentamicina, esta fue inferior al 65,0\%, mientras que para amikacina fue inferior al 15,0\% en ambos microorganismos.

Tabla 1. Prevalencia de aislamientos de $E$. coli y $K$. pneumoniae resistentes a cefalosporinas de tercera generación $\left(C-3^{\mathrm{a}} G\right)$ en las cuatro instituciones de salud participantes

\begin{tabular}{c|cccc}
\hline Microorganismo & Institución & $\begin{array}{c}\text { No. total, de } \\
\text { aislamientos }\end{array}$ & $\begin{array}{c}\text { No. de aislamientos resistente a } \\
\mathbf{C - 3} \mathbf{G}\end{array}$ & $\begin{array}{c}\text { Prevalencia } \\
\text { \% }\end{array}$ \\
\hline \multirow{5}{*}{ E. coli. } & $\mathrm{A}$ & 1554 & 220 & 14,1 \\
\cline { 2 - 5 } & $\mathrm{B}$ & 694 & 179 & 25,8 \\
& $\mathrm{C}$ & 453 & 111 & 24,5 \\
\cline { 2 - 5 } & $\mathrm{D}$ & 859 & 325 & 37,8 \\
& Total & $\mathbf{3 5 6 0}$ & $\mathbf{8 3 5}$ & $\mathbf{2 3 , 5}$ \\
\hline \multirow{5}{*}{ K. pneumoniae. } & $\mathrm{A}$ & 582 & 93 & 16,0 \\
& $\mathrm{~B}$ & 225 & 48 & 21,3 \\
\cline { 2 - 5 } & $\mathrm{C}$ & 204 & 80 & 39,2 \\
& $\mathrm{D}$ & 543 & 194 & $\mathbf{2 6 , 7}$ \\
\cline { 2 - 5 } & Total & $\mathbf{1 5 5 4}$ & $\mathbf{4 1 5}$ & \\
\hline
\end{tabular}




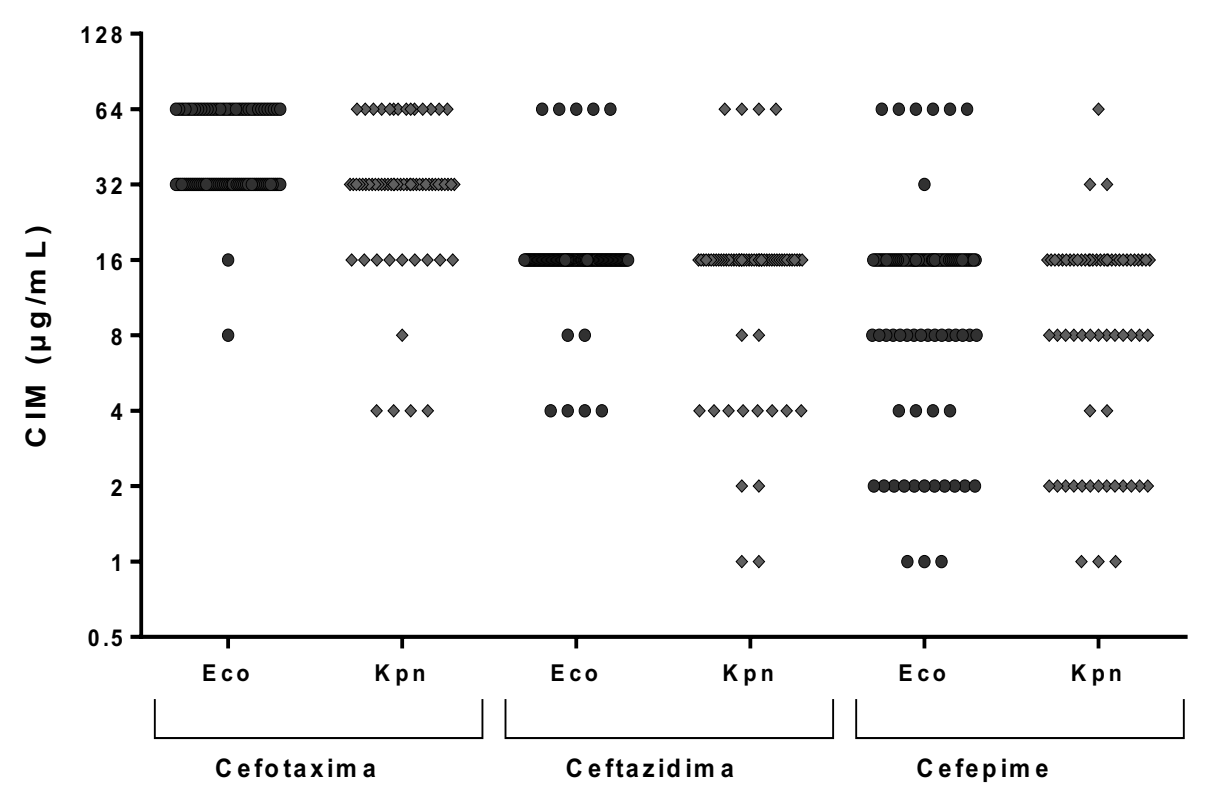

Figura 1. Distribución de la concentración inhibitoria mínima (CIM $\mu \mathrm{g} / \mathrm{mL}$ ), para cefotaxima (resistente $\geq 4 \mu \mathrm{g} / \mathrm{mL}$ ), ceftazidima (resistente $\geq 16 \mu \mathrm{g} / \mathrm{mL}$ ) y cefepime (resistente $\geq 16 \mu \mathrm{g} / \mathrm{mL} \mu \mathrm{g} / \mathrm{mL}$ ) en los aislamientos de Escherichia coli (Eco) y K. pneumoniae (Kpn) recuperados durante el periodo de estudio.

\section{Caracterización fenotípica}

La información obtenida de los 164 aislamientos analizados permitió agrupar el 91\% de las E. coli en tres perfiles, mientras que el $85,93 \%$ de K. pneumoniae se agrupo en cuatro perfiles (Tabla 2).

Tabla 2. Perfiles fenotípicos de resistencia a $C-3^{\mathrm{a}} \mathrm{G}$ y su resistencia acompañante en $E$. coli y $K$. pneumoniae

\begin{tabular}{|c|c|c|c|c|c|c|c|c|}
\hline \multirow[t]{2}{*}{ Características } & & \multicolumn{3}{|c|}{$\begin{array}{c}\text { E. coli } \\
\text { Perfiles * }\end{array}$} & \multicolumn{4}{|c|}{$\begin{array}{l}\text { K. pneumoniae } \\
\text { Perfiles * }\end{array}$} \\
\hline & & $\mathrm{I}(\mathrm{n}=60)$ & II $(n=28)$ & III $(n=3)$ & $\mathrm{I}(\mathrm{n}=10)$ & II $(n=31)$ & III (n=11) & IV $(n=3)$ \\
\hline \multirow{2}{*}{ C-3 $\mathbf{3}^{\mathbf{a}} \mathbf{G} \mathbf{C I M}(\mu \mathrm{g} / \mathrm{mL})$} & CTX & $>=32$ & $>=64$ & $>=64$ & $>=4<=16$ & $>=32$ & $>=64$ & $>=64$ \\
\hline & CAZ & $>=16$ & $>=16$ & $>=64$ & $>=4<=16$ & $>=16$ & $>=16$ & $>=64$ \\
\hline Prueba D-D & D-D + & 57 & 23 & 3 & 7 & 26 & 11 & 2 \\
\hline \multirow{5}{*}{$\begin{array}{l}\text { Resistencia a otros } \\
\beta \text {-lactámicos (n) }\end{array}$} & FOX & 0 & 1 & 1 & 0 & 6 & 0 & 1 \\
\hline & $\mathrm{TZP}$ & 6 & 2 & 0 & 3 & 12 & 1 & 2 \\
\hline & FEP & 58 & 28 & 3 & 9 & 30 & 11 & 3 \\
\hline & ETP & 3 & 0 & 0 & 0 & 5 & 0 & 2 \\
\hline & MEM & 0 & 0 & 0 & 0 & 2 & 0 & 1 \\
\hline \multirow{4}{*}{$\begin{array}{l}\text { Resistencia a otras } \\
\text { familias de antibióticos } \\
\text { (n) }\end{array}$} & GEN & 35 & 13 & 1 & 7 & 20 & 8 & 2 \\
\hline & AMK & 2 & 3 & 0 & 2 & 5 & 0 & 0 \\
\hline & CIP & 54 & 26 & 3 & 6 & 21 & 5 & 0 \\
\hline & SXT & 51 & 25 & 3 & 9 & 22 & 10 & 3 \\
\hline
\end{tabular}

C-3 ${ }^{\mathrm{a}} \mathrm{G}$ : cefalosporinas de tercera generación; CTX: cefotaxima; CAZ: ceftazidima; FOX: cefoxitin; TZP: piperacilina tazobactam; FEP: Cefepime; ETP: ertapenem; MEM: meropenem; GEN: gentamicina; AMK: amikacina; CIP: ciprofloxacina; SXT: trimetoprim- sulfametoxazol.

* Estos perfiles están definidos por la CIM de CTX/CAZ y las características asociadas como prueba de doble disco, genes de $\beta$-lactamasas de espectro extendido, resistencia a otros $\beta$-lactámicos y resistencia a otras familias de antibióticos. 
Cabe resaltar que el perfil-I de E. coli (CIM: 16 - 32 $\mu \mathrm{g} / \mathrm{mL}$ para cefotaxima/ceftazidima), además de agrupar el mayor número de aislamientos con pruebas de D-D positiva $(57 / 60)$, se presentó resistencia a cefepime del 96,6\% (CIM $\geq 16 \mu \mathrm{g} / \mathrm{mL})$, sensibilidad a piperacilina tazobactam del $90 \%$ $(\mathrm{CIM} \leq 8 / 4 \mu \mathrm{g} / \mathrm{mL})$ y una variada resistencia a otras familias de antibióticos, siendo ciprofloxacina y trimetoprim-sulfametoxazol los antibióticos más afectados con un $90 \%$ y $85 \%$ respectivamente (Tabla $3)$. Por otro lado, el $45 \%$ de las E. coli con perfil-I provenían del servicio de urgencias, el que aportó mayor número de aislamientos (Tabla 3 ).
El perfil - II de K. pneumoniae agrupó 31 cepas, de las cuales el 83,87\% (26/31) dio una prueba de doble disco positiva. Además, la resistencia a cefepime fue mayor al $90 \%$ y la resistencia a otras familias de antibióticos fue variada, siendo el trimetoprimsulfametoxazol el más afectado con un 70,96\% (22/31) (Tabla 2). El 41,9\% de los aislamientos dentro de este perfil fue aportado por una institución y provenían de los servicios de hospitalización y UCI.

Tabla 3. Distribución de los perfiles de resistencia por institución y servicio hospitalario

\begin{tabular}{|c|c|c|c|c|c|c|c|}
\hline \multirow{2}{*}{ Microorganismo } & \multirow{2}{*}{ Perfil } & \multirow{2}{*}{ Institución } & \multicolumn{5}{|c|}{ No. de aislamientos por servicio } \\
\hline & & & C. Ext & Urg & Hosp & UCI & Total \\
\hline \multirow{7}{*}{ E. coli } & \multirow{3}{*}{$\begin{array}{c}\mathrm{I} \\
(\mathrm{n}=60)\end{array}$} & $\mathrm{A}$ & 1 & 8 & 10 & 1 & 20 \\
\hline & & $\mathrm{B}$ & 12 & 9 & 2 & 4 & 27 \\
\hline & & $\mathrm{C}$ & 2 & 6 & 4 & 1 & 13 \\
\hline & \multirow{3}{*}{$\begin{array}{c}\mathrm{II} \\
(\mathrm{n}=28)\end{array}$} & $\mathrm{A} / \mathrm{C}$ & 0 & 2 & 0 & 0 & 2 \\
\hline & & $\mathrm{B}$ & 3 & 3 & 2 & 0 & 8 \\
\hline & & $\mathrm{D}$ & 9 & 3 & 4 & 2 & 18 \\
\hline & $\begin{array}{c}\text { III } \\
(n=3)\end{array}$ & $\mathrm{D}$ & 0 & 1 & 1 & 1 & 3 \\
\hline \multirow{10}{*}{ K. pneumoniae } & \multirow{3}{*}{$\mathrm{I}(\mathrm{n}=10)$} & $\mathrm{A}$ & 0 & 0 & 0 & 1 & 1 \\
\hline & & $\mathrm{C}$ & 0 & 0 & 5 & 0 & 5 \\
\hline & & $\mathrm{D}$ & 0 & 2 & 0 & 2 & 4 \\
\hline & \multirow{3}{*}{$\begin{array}{c}\text { II } \\
(n=31)\end{array}$} & $\mathrm{A}$ & 0 & 0 & 5 & 6 & 11 \\
\hline & & B & 1 & 2 & 4 & 0 & 7 \\
\hline & & $\mathrm{C}$ & 0 & 1 & 6 & 6 & 13 \\
\hline & \multirow{2}{*}{$\begin{array}{c}\text { III } \\
(n=11)\end{array}$} & $\mathrm{A}$ & 0 & 0 & 0 & 1 & 1 \\
\hline & & D & 2 & 0 & 5 & 3 & 10 \\
\hline & \multirow{2}{*}{$\operatorname{IV}(n=3)$} & $\mathrm{B}$ & 0 & 0 & 1 & 0 & 1 \\
\hline & & $\mathrm{D}$ & 0 & 1 & 0 & 1 & 2 \\
\hline
\end{tabular}

C. Ext: consulta externa; Urg: urgencias; Hosp: hospitalización diferente a UCI; UCI: unidad de cuidados intensivos.

Relación entre la prueba de doble disco y la CIM El porcentaje total de aislamientos positivos para la prueba de doble disco (D-D) fue 90,2\% (148/164); de estos, el 91\% se observó en E. coli (91/100) y $87,5 \%$ (56/64) en $\mathrm{K}$. pneumoniae. El diámetro promedio de una prueba D-D positiva para E. coli fue de $13,88 \mathrm{~mm}$ (IC 95\%: 12,75 - 15,05) y para K. pneumoniae fue de 14,78 mm (IC 95\%: 12,98 -
16,59). Valores de CIM mayores o iguales a 16 $\mu \mathrm{g} / \mathrm{mL}$ estuvieron asociados con valores positivos de una prueba D-D. De igual forma para ceftazidima, solo cuando se reportaron CIM mayores o iguales a $16 \mu \mathrm{g} / \mathrm{mL}$ se observó una prueba D-D positiva con valores superiores a $8 \mathrm{~mm}$. 


\section{DISCUSIÓN}

De acuerdo a los resultados fenotípicos de BLEE arrojados por nuestro estudio, se evidenció una prevalencia de $91 \%$ en E. coli $(91 / 100)$ y del $87,5 \%$ (56/64) en K. pneumoniae, con una resistencia a la ciprofloxacina de $80 \%$ y $78.1 \%$ respectivamente, datos que contrastan con lo expresado por Villegas en un estudio realizado en las UCI de once instituciones de salud de Colombia, encontrando para E. coli entre $10-13 \%$ y K. pneumoniae un $30 \%$, y una resistencia del $20-40 \%$ a ciprofloxacina (21). En otro estudio realizado por Villegas et al, en el 2004, se indicó que esta prevalencia se encontraba por encima del $40 \%$ en los hospitales de Colombia (22); pero, en un estudio de Leal et al del 2013, se reportaron fenotipos de resistencia a cefalosporinas de tercera generación que variaron entre el 3,4\% y $6,3 \%$ para E. coli y $3,4 \%$ y $17,2 \%$ para K. pneumoniae, todos procedentes de infecciones de tracto urinario de origen comunitario (12).

En nuestro estudio la prevalencia promedio de resistencia a cefalosporinas de tercera generación reportada por las instituciones participantes fue de $23,5 \%$ para E. coli y de $26,7 \%$ para K. pneumoniae, resultados similares al estudio realizado en el 2017 por Giovanetti et al, en instituciones en salud del departamento del Cesar, Colombia, donde registraron resistencia para K. pneumoniae y E. coli en aislados en UCI con una prevalencia del $28.3 \%$ y del $21.8 \%$ respectivamente, y para servicios diferentes a UCI presentaron una resistencia del $43.1 \%$ para K. pneumoniae y del $17 \%$ para E. coli (23). Villalobos et al, reportaron resistencia en E. coli del $20 \%$ y $35,6 \%$ en K. pneumoniae, porcentajes que para la E. coli serían muy semejantes a nuestro resultado, aunque no para la K. pneumoniae, llamando la atención esta prevalencia (24). En otro estudio realizado en el 2015 por Gómez JF y Sánchez JA, en una UCI de la ciudad de Pereira, Colombia, se evidenció la presencia de bacterias BLEE, con el $32 \%$ para E. coli y el $16 \%$ para $\mathrm{K}$. pneumoniae, observándose que, aunque estas prevalencias son diferentes, no están muy alejadas a los valores de nuestro estudio (25). Por otro lado, en un estudio de resistencia antimicrobiana realizado en el 2018 por Ibáñez et al, en aislamientos urinarios de pacientes en un servicio de urgencia de Manizales, Colombia, los microorganismos más frecuentemente aislados fueron E. coli y K. pneumoniae, con un porcentaje de resistencia a cefalosporinas de tercera generación del $14 \%$ y $22 \%$ respectivamente, evidenciándose que el porcentaje de K. pneumoniae, es cercano al evidenciado en nuestro estudio (26). Aunque en la región Caribe colombiana la información de la resistencia bacteriana es limitada, en el 2003 Martínez et al, realizaron un estudio en tres hospitales de la Costa Atlántica, reportando una prevalencia de BLEE en E. coli que osciló entre 17,6 $\%$ y $20,5 \%$ y para $\mathrm{K}$. pneumoniae entre $40 \%$ y $46 \%$ (27), dato muy cercano a nuestro estudio con relación a E. coli. En ese mismo año, un estudio realizado por Villanueva et al en dos hospitales reportó a K. pneumoniae y E. coli como productoras de BLEE en un $40 \%$ y $17.6 \%$ respectivamente (28).

Los datos de estudios mencionados, se encuentran cercanos a los datos obtenidos en nuestro estudio para uno u otro microorganismo, lo que refleja la necesidad de implementar y estandarizar la vigilancia de estos eventos, tanto a nivel local como a nivel nacional, lo cual permitirá fortalecer la vigilancia de estos microorganismos.

Por otro lado, nuestros datos reflejan importantes diferencias epidemiológicas entre los dos microrganismos estudiados, mientras que el $63 \%$ de las E. coli provenían de servicios de urgencias y consulta externa ( $70 \%$ aisladas de orina), el $80 \%$ de las K. pneumoniae se aislaron de los servicios de UCI y hospitalizados (39\% sangre y $13 \%$ tracto respiratorio), lo que sugiere la posibilidad de que E. coli productora de BLEE y multiresistente a cefepime, trimetoprim- sulfametoxazol, ciprofloxacina y gentamicina esté circulando a nivel comunitario en nuestra ciudad (Tabla 2); sin embargo, no se puede afirmar que estos pacientes no hayan ingresado previamente a las instituciones hospitalarias o hayan estado expuestos a antimicrobianos, por lo que estos hallazgos deben ser corroborados por estudios clínicos que determinen su origen. Todas las cepas BLEE analizadas mostraron altos porcentajes de resistencia 
a trimetoprim-sulfametoxazol, ciprofloxacina y gentamicina, sin embargo, se mantienen con alta sensibilidad a los carbapenemes, piperacilinatazobactam y amikacina (Tabla 2). Hallazgos similares han determinado que la sensibilidad de las enterobacterias productoras de BLEE para cefepime, fluoroquinolonas, ampicilina/sulbactam y aminoglucósidos ha disminuido notablemente, dejando a los carbapenémicos, tigeciclina y colistina como los antibióticos de elección $(29,30)$.

Aunque E. coli tuvo predominio en los servicios de urgencias y consulta externa, un $10 \%$ de estas cepas estuvo asociado a UCI, por lo que esta cifra llama la atención ya que la morbimotalidad puede ser alta en este servicio. En nuestro país, estudios sobre la evolución de la resistencia en UCI han reportado que E. coli es un microrganismo frecuente (14). En Latinoamérica, por ejemplo, en Perú, el $60 \%$ de los aislados de UCI en un hospital de alta complejidad durante un año correspondieron a E. coli productora de BLEE (31); mientras que, en Brasil, datos de la agencia ANVISA indicaron que E. coli estuvo involucrada en cerca del $29 \%$ de las infecciones en la UCI adultos, y en la UCI neonatal las tasas estuvieron cercanas al 17\% (32).

En conclusión, observamos que en la ciudad de Barranquilla los fenotipos de BLEE circulan en varias instituciones y servicios hospitalarios y se acompañan de multirresistencia a cefepime, trimetoprim-sulfametoxazol, ciprofloxacina y gentamicina, sin embargo, aún mantienen alta sensibilidad a los carbapenemes, piperacilinatazobactam y amikacina. La divulgación periódica de estos fenotipos de resistencia circulantes en nuestras instituciones de salud genera información útil para la selección de las opciones terapéuticas adecuadas; esto confirma una vez más, la necesidad de fortalecer las acciones de prevención y control para evitar su diseminación en la población.

\section{AGRADECIMIENTOS}

A las cuatro instituciones de salud de la ciudad de Barranquilla, Colombia, que aportaron los aislamientos y la información necesaria para el desarrollo del proyecto. A la Universidad
Metropolitana por la financiación de esta investigación.

CONFLICTOS DE INTERESES: Los autores declaran que no hay conflicto de intereses.

FINANCIACIÓN: El proyecto fue financiado con recursos aportados por la Universidad Metropolitana de Barranquilla.

\section{REFERENCIAS}

1. Barriere SL. Clinical, economic and societal impact of antibiotic resistance. Expert Opin Pharmacother. 2015;16:151-3.

http://dx.doi.org/10.1517/14656566.2015.983077

2. Smith R, Coast J. The true cost of antimicrobial resistance. BMJ. 2013;346: f1493-7. http://researchonline.1shtm.ac.uk/660655/

3. Calderón G, Aguilar L. Resistencia antimicrobiana: Microorganismos más resistentes y antibióticos con menor actividad. Revista Médica de Costa Rica y Centroamérica. 2016;(621):757 - 763.

4. Temkin E, Fallach N, Almagor J, Primrose B, Tacconelli E, Carmel Y. Estimating the number of infections caused by antibiotic-resistant Escherichia coli and Klebsiella pneumoniae in 2014: a modelling study. The Lancet Global Health. 2018;(6):969-979. https://doi.org/10.1016/S2214-109X(18)30278-X

5. Villalobos AP, Barrero LI, Rivera SM, Ovalle Valera MV, Valera Danik. Vigilancia de infecciones asociadas a la atención en salud, resistencia bacteriana y consumo de antibióticos en hospitales de alta complejidad, Colombia, 2011. Biomédica 2014;34 Supl1:67-80. http://dx.doi.org/10.7705/biomedica.v34i0.1698

6. Rodríguez-Vidigal FF, Vera-Tomé A, NogalesMuñoz N, Muñoz-Sanz A. Infecciones por enterobacterias productoras de betalactamasas de espectro extendido tras cirugía cardiaca: su impacto en la mortalidad. Revista Colombiana de Cardiología. 2015. http://dx.doi.org/10.1016/j.rccar.2015.10.006

7. López-Velandia DP, Torres-Caycedo MI, PradaQuiroga CF. Genes de resistencia en bacilos Gram negativos: Impacto en la salud pública en Colombia. Rev Univ. Salud. 2016;18(1):190-202. 
8. Ovalle M, Saavedra S, González M, Hidalgo A, Duarte C, Beltrán M. Resultados de la vigilancia nacional de la resistencia antimicrobiana de enterobacterias y bacilos Gram negativos no fermentadores en infecciones asociadas a la atención de salud, Colombia, 2012-2014. Biomédica 2017;37:473-85. https://doi.org/10.7705/biomedica.v34i2.3432

9. Morejón-García M. Betalactamasas de espectro extendido. Revista Cubana de Medicina. 2013;52(4):272280.

http://scielo.sld.cu/scielo.php?script $=$ sci arttext\&pid $=\mathrm{S} 0$ $\underline{034-75232013000400006 \& \operatorname{lng}=\mathrm{es}}$

10. Jiménez A, Alvarado A, Gomez F, Carrero G, Fajardo C. Factores de riesgo asociados al aislamiento de Escherichia coli o Klebsiella pneumoniae productoras de betalactamasas de espectro extendido en un hospital de cuarto nivel en Colombia. Biomedica. 2014;34 Supl1:1622. http://dx.doi.org/10.7705/biomedica.v34i0.1650

11. Cifuentes M, Silva F, Arancibia JM, Rosales R, Ajenjo MC, Riedel G, et al. Grupo Colaborativo de Resistencia Bacteriana, Chile: recomendaciones 2014 para el control de la resistencia bacteriana. Rev Chilena Infectol. 2015; 32(3):305-318. http://dx.doi.org/10.4067/S0716-10182015000400008

12. Gonzalez L, Cortes JA. Revisión sistemática de la farmacorresistencia en enterobacterias de aislamientos hospitalarios en Colombia. Biomedica. 2014;34:180-97. http://dx.doi.org/10.7705/biomedica.v34i2.1550

13. Dokyun K, Ji Young A, Chae Hoon L, Sook J, Hyukmin L, Dongeun Y et al. Increasing Resistance to Extended-Spectrum Cephalosporins, Fluoroquinolone, and Carbapenem in Gram-Negative Bacilli and the Emergence of Carbapenem Non-Susceptibility in Klebsiella pneumoniae: Analysis of Korean Antimicrobial Resistance Monitoring System (KARMS) data from 2013 to 2015. Ann Lab Med 2017;37:231-239. https://doi.org/10.3343/alm.2017.37.3.231

14. Hernández-Gómez $\mathrm{C}$, Blanco VM, Motoa G, Correa A, Maya JJ, Cadena E et al. Evolución de la resistencia antimicrobiana de bacilos Gram negativos en unidades de cuidados intensivos en Colombia. Biomedica. 2014;34 Supl1:91-100. http://dx.doi.org/10.7705/biomedica.v34i0.1667

15. Leal AL, Cortés JA, Arias G, Ovalle MV, Saavedra $\mathrm{SY}$, Buitrago $\mathrm{G}$, et al. Emergencia de fenotipos resistentes a cefalosporinas de tercera generación en
Enterobacteriaceae causantes de infección del tracto urinario de inicio comunitario en hospitales de Colombia. Enfermedades Infecciosas y Microbiología Clínica. 2013;31:298-303

http://dx.doi.org/10.1016/j.eimc.2012.04.007.

16. Instituto Nacional de Salud (INS). Resultados del Programa de Informe de Resultados de Vigilancia por Laboratorio de Resistencia antimicrobiana en Infecciones Asociadas a la Atención en Salud (IAAS) 2018. Disponible en: https://www.ins.gov.co/buscadoreventos/Informacin $\% 20 \mathrm{de} \% 20$ laboratorio/Informe $\% 20$ Vigilancia\%20por\%20Laboratorio\%20Resistencia\%20 Antimicrobiana $\% 20 \mathrm{y} \% 20$ Whonet $\% 20 \mathrm{IAAS} \% 202017 . \mathrm{p}$ df

17. Clinical and Laboratory Standards Institute. Performance Standards for Antimicrobial Susceptibility Testing: Twenty-Four Informational Supplement. CLSI document M100-S24. Wayne, PA: Clinical and Laboratory Standards Institute; 2014.

18. Clinical and Laboratory Standards Institute. Performance Standards for Antimicrobial Susceptibility Testing: Twenty-Five Informational Supplement. CLSI document M100-S25. Wayne, PA: Clinical and Laboratory Standards Institute; 2015.

19. Pacheco R, Osorio L, Correa AM, Villegas MV. Prevalencia de bacterias Gram negativas portadoras del gen blaKPC en hospitales de Colombia. Biomédica2014;34(Sup1.1):81-90. http://dx.doi.org/10.7705/biomedica.v34i0.1642

20. Cavalieri S, Harbeck R, McCarter Y, Ortez J, Rankin I, Sautter R et al. Manual de pruebas de susceptibilidad antimicrobiana. 2005. https://www.paho.org/hq/dmdocuments/2005/susceptibil idad-antimicrobiana-manual-pruebas-2005.pdf

21. Villegas MV. Epidemiology of nosocomial Gram () bacteria in Colombia: an update. 3rd International Symposium on Antimicrobial Resistance, Cartagena; 2004.

22. Villegas M, Correa A, Pérez F, Miranda M, Zuluaga T, Quinn J. Colombian Nosocomial Resistance Study Group. Prevalence and characterization of extendedspectrum beta-lactamases in Klebsiella pneumoniae and Escherichia coli isolates from Colombian hospitals. Diagn Microbiol Infect Dis. 2004;49:217-22. http://dx.doi.org/10.1016/j.diagmicrobio.2004.03.001 
23. Giovanetti Y, Morales-Parra GI, Armenta-Quintero C. Perfil de resistencia bacteriana en hospitales y clínicas en el departamento del Cesar (Colombia). Medicina\& Laboratorio 2017; 23:387-398http://docs.bvsalud.org/biblioref/2018/05/883698/resiste ncia-bacteriana.pdf

24. Villalobos Rodríguez AP, Díaz Ortega MH, Barrero Garzón LI, Rivera Vargas SM, Henríquez Iguarán DE, Villegas Botero MV, et al. Tendencias de los fenotipos de resistencia bacteriana en hospitales públicos y privados de alta complejidad de Colombia. Revista Panamericana de Salud Pública. 2011;30:627-633. http://dx.doi.org/10.1590/S1020-49892011001200022

25. Gómez-González JF, Sánchez-Duque JA. Perfil microbiológico y resistencia bacteriana en una unidad de cuidados intensivos de Pereira, Colombia, 2015. MÉD. UIS. 2018;31(2):9-15. Páginas. DOI: 10.18273/revmed.v31n2-2018001

26. Ibañez-Dosman JP et al. Panorama de resistencia antimicrobiana de los aislamientos urinarios de pacientes adultos en los servicios de urgencias de Manizales, Caldas, durante el 2018. Infectio 2020; 24(3):149-154. http://www.scielo.org.co/pdf/inf/v24n3/0123-9392-inf24-03-149.pdf

27. Martínez P, Mercado M, Máttar S. Determinación de B-lactamasas de espectro extendido en gérmenes nosocomiales del Hospital San Jerónimo, Montería. Colombia Médica. 2003;34:196-205. http://colombiamedica.univalle.edu.co/index.php/comed ica/article/view/274/277.
28. Villanueva A, Martínez P, Máttar S, Urbina D. Prevalencia de $\beta$-Lactamasas de espectro extendido (BLEE) en el Hospital San Jerónimo de Montería y la Clínica General del Norte de Barranquilla. Infectio 2003; 7:103

29. Quiñones D, Carmona Y, Zayas A, Abreu M, Salazar $\mathrm{D}$, García $\mathrm{S}$, et al. Resistencia antimicrobiana en aislamientos clínicos de Klebsiella spp. y producción de B-lactamasas de spectro extendido en hospitales de Cuba. Revista Cubana de Medicina Tropical. 2014;66(3):386$399 . \quad$ Disponible en: http://scielo.sld.cu/scielo.php?script=sci arttext\&pid=S0 $\underline{375-07602014000300007 \& \operatorname{lng}=\text { es }}$

30. Abreu S, Varela Y, Millán B, Araque, M. Klebsiella pneumoniae y Escherichia coli productoras de betalactamasas de espectro extendido, aisladas en pacientes con infección asociada a los cuidados de la salud en un hospital universitario. Enfermedades Infecciosas y Microbiología. 2014;34 (3): 92-99.

31. Guzmán-Blanco M, Labarca JA, Villegas MV, Gotuzzo E. Extended spectrum $\beta$-lactamase producers among nosocomial Enterobacteriaceae in Latin America. Brazilian Journal of Infectious Diseases. 2014;18:421-33. http://dx.doi.org/10.1016/j.bjid.2013.10.005.

32. Patrick DM, Chambers C, Purych D, Chong M, George D, Marra F. Value of an aggregate index in describing the impact of trends in antimicrobial resistance for Escherichia coli. Can J Infect Dis Med Microbiol. 2015;26: p. 33-8. 